\title{
Against the Complex versus Simple Distinction
}

\author{
Patrik Hummel ${ }^{1}$
}

Received: 13 September 2015/Accepted: 21 May 2016/Published online: 7 June 2016

(C) The Author(s) 2016. This article is published with open access at Springerlink.com

\begin{abstract}
This paper examines three proposals on the difference between the complex and the simple view about personal identity: Parfit's original introduction of the distinction, Gasser and Stefan's definition, and Noonan's recent proposal. I argue that the first two classify the paradigm cases of simplicity as complex, while Noonan's proposal makes simplicity and complexity turn on features whose relevance for the distinction is questionable. Given these difficulties, I examine why we should be interested in whether a position is complex or simple. I describe two purposes of having a distinction, and show that extant accounts of the complex versus simple distinction fail to serve these. I argue that unless we find a satisfying account of the difference between complex and simple positions, we should not frame discourses on personal identity in these terms.
\end{abstract}

\section{Introduction}

As observers and disputants, we face choices about the theoretical tools by means of which we think about a given subject. Sometimes, new concepts or distinctions advance discussion greatly. But there are other times when utilization of a concept or distinction blurs disputes or confuses us. The present paper argues that the distinction between the complex and the simple view of personal identity is of this kind.

Debates on personal identity are often framed in terms of this distinction, and the literature acknowledges clear paradigm cases of either side (Parfit 1982: 227; Garrett 2006: 122; Noonan 2011: 72). I examine three proposals on what the

Patrik Hummel

pah20@st-andrews.ac.uk

1 Arché, University of St Andrews, 17 - 19 College Street, St Andrews, Fife KY16 9AL, Scotland, UK 
difference between the complex and the simple view is: Parfit's introduction of the terms (Sect. 2), Gasser and Stefan's definition (Sect. 3), and Noonan's recent proposal (Sect. 4). I argue that given their shortcomings, the complex versus simple distinction fails to serve two important purposes of having a given distinction in our discourse: the facilitation of classification and endorsement of positions (Sect. 5). We thus should not frame discourses in terms of the complex versus simple distinction.

Throughout this paper, I will use the term 'view' as denoting what unites a family of positions, where a 'position' is a particular instance of a view. For example, Shoemaker's position is an instance of the complex view.

\section{Parfit's Definition}

One straightforward way to investigate the difference between the complex and the simple view is to look in the place where the distinction is drawn for the first time.

\subsection{Introduction of Complex/Simple and Reductionism/Non-reductionism}

Parfit defines:

"According to the Complex View, the fact of personal identity over time just consists in the holding of certain other facts. It consists in various kinds of psychological continuity, of memory, character, intention, and the like, which in turn rest upon bodily continuity. According to the Simple View, personal identity does not just consist in these continuities, but is a quite separate 'further fact.", (Parfit 1982: 227)

In subsequent writings, Parfit drops the terms 'complex' and 'simple' without further announcement, and distinguishes reductionism from non-reductionism. Reductionists believe

“(1) that the fact of a person's identity over time just consists in the holding of certain more particular facts, and

(2) that these facts can be described without either presupposing the identity of this person, or explicitly claiming that the experiences in this person's life are had by this person, or even explicitly claiming that this person exists. These facts can be described in an impersonal way." (Parfit 1984: 210)

In contrast,

"[o]ur view is Non-Reductionist if we reject both of the two Reductionist claims." (Parfit 1984: 210)

This rejection can happen in one of the following ways (ibid.): by claiming that persons are

- separately existing entities, distinct from brains, bodies and experiences, for example Cartesian egos; 
- not separately existing entities, yet their existence is a further fact which does not just consist in physical and/or psychological continuity.

\subsection{The Relation Between Complex/Simple and Reductionism/Non- reductionism}

The relation between the complex view and reductionism on the one hand, and between the simple view and non-reductionism on the other, is not entirely clear. Parfit (1982: 227) says that 'complex' and 'simple' are

"shorter labels for the two main views [reductionism and non-reductionism]," but also explains:

"The central claims [my emphasis] of the reductionist tradition I shall call the Complex View, those of the non-reductionist tradition the Simple View."

This leads some interpreters to regard the complex view and reductionism as equivalent (Wasserman 2012: Fn. 1). Others think the complex view is a forerunner to reductionism (Zimmerman 2012: 206). Others think reductionism is a more specific doctrine than the complex view (Noonan 2003: Ch. 5.6.) because of clause (2): the complex view is neutral on whether the more particular facts can be described without mentioning persons; Parfit's reductionism explicitly claims this.

So much seems clear: even though Parfit did not continue to talk in these terms, acceptance of the complex view is what unites the different reductionist positions, and the same goes for the simple view and non-reductionism. But he is not explicit on how much overlap there is between the complex view and reductionism and the simple view and non-reductionism respectively, for example whether they share their opinions solely on (1) or on (2) as well. For what follows, the answer does not really matter.

\subsection{Misclassifications}

Parfit (1982: 227) mentions paradigm cases of either side: Hume, Locke, Lewis and Parfit himself accept the complex view, Butler, Reid, Chisholm and Swinburne endorse the simple view.

This, I argue, should strike us as puzzling. The supposed paradigm cases of simplicity do not satisfy Parfit's definition. While Butler (1736: 100), Reid (1785a: 108-109) and Swinburne (1984: 18-20) are notoriously dismissive of positive stories about personal identity, they do operate against the backdrop of a Cartesian or Leibnizian metaphysics, and so I agree with Olson (2012: 49-50) and disagree with Duncan (2014: 284-285) that nothing in their works precludes acceptance of claims like the following:

- person $\mathrm{x}$ at $\mathrm{t}$ is the same person as $\mathrm{y}$ at $\mathrm{t}^{*}$ iff $\mathrm{x}$ and $\mathrm{y}$ have the same ego or soul (given Swinburne 1984: 21); 
- person $\mathrm{x}$ at $\mathrm{t}$ is the same person as $\mathrm{y}$ at $\mathrm{t}^{*}$ iff $\mathrm{x}$ is a monad, $\mathrm{y}$ is a monad, and $\mathrm{x}$ is the same monad as y (given Reid 1785b: 109).

If so, they satisfy (1): a person's identity over time consists in more particular facts, namely that a certain soul or ego exists at the relevant times.

In Reid's case, there are two reasons why one might deny that (1) is satisfied. Firstly, Reid claims that persons are identical to monads, not just that personal identity consists in monad identity. Secondly, given that persons are identical to monads, facts about monads are not more particular than facts about persons. In response, I am prepared to accept that some simple positions do not satisfy (1). What matters for my purposes is that there are simple positions which definitely make consist-in claims and thus satisfy (1). Moreover, I deny that because of his identity claim, Reid should be seen as rejecting (1). Parfit explicitly includes positions that make identity claims between persons and other things under the umbrella of reductionism; he even calls them "hyper-Reductionist" (1995: 16). In addition, positions according to which persons are identical to collections of matter or brains or biological organisms are certainly instances of the complex view (Gasser and Stefan 2012: 4-7). If these positions count as satisfying (1) by reducing facts about persons to more particular facts, then I see no reason why Reid could not do the same.

Moreover, authors like Swinburne and Reid can even satisfy (2): they can accept that the facts mentioned in (1) can be described in an impersonal way, without presupposing the existence or persistence of persons, for example by referring solely to egos, souls or monads. I claim that this holds even for Reid and others who think that persons are identical to these entities. This suspicion is motivated by the observation that no one takes a philosopher who believes that persons are identical to collections of material particles as being unable to provide an impersonal description of the world. In support, assume that persons are identical to collections of human-wise arranged, material particles. Suppose also that I can describe the world exhaustively in terms of particle distribution. When describing the world, do I have to presuppose that persons exist? Of course, I can assume in advance that persons exist, and make my particle story reflect this. But I could also stay antecedently neutral on questions regarding persons, and proceed to tell you the exact distribution of each particle in a given universe. From this, it might or might not then follow that persons exist and persist. But if it does, it will not have something I have presupposed before making my description. And I suppose that exactly the same should apply to a philosopher who chooses to describe the world not in terms of particles, but in terms of monads, souls or egos. I am not claiming that there are passages in, e.g., Reid's writings which clearly suggest this reasoning. I also do not claim that he must follow this reasoning. But I do maintain that this reasoning is at least open to him and other simple positions.

Again, I do not claim that all simple positions accept (1) and (2). What matters for my purposes is that there are paradigm cases of simplicity which leave room for joint satisfaction of (1) and (2) and therewith satisfy the conditions for being complex. I conclude that Parfit has introduced a distinction, but not one that captures the paradigm cases. 


\section{Gasser and Stefan}

One might hope that our grasp of the distinction has improved in the meantime. Gasser and Stefan in their volume on the complex versus simple distinction offer the following summarizing definitions:

"The complex view analyzes personal identity in terms of simpler relations. The fact that a person persists over time is nothing more than some other facts which are generally spelled out in either biological or psychological terms, or both. That is, the complex view takes talk about what personal identity consists in literally. It aims to provide necessary and sufficient conditions for personal identity, thereby reducing it to the holding of basic biological or psychological relations. Whenever these relations obtain, personal identity obtains.

The simple view of personal identity, by contrast, denies that a person's identity through time consists in anything but itself. Biological and psychological continuity may be regarded as epistemic criteria for diachronic identity, but they are neither necessary nor sufficient conditions for personal identity. There are no non-circular, informative necessary and sufficient conditions for personal identity: personal identity consists in nothing other than itself" (Gasser and Stefan 2012: 3).

These definitions shift between different notions that are supposed to mark the difference: consisting in something other than itself, non-epistemic criteria for identity, definability, analysability, non-circularity, informativeness, necessary and sufficient conditions. These notions are certainly connected, but the connection is not straightforward enough to shift between them without further explanation. I will discuss them in turn.

Before I do this, let me add one clarification. By accepting the complex view as just defined, one need not accept that identity as such is analysable, or consists in other relations which are necessary and sufficient for its obtainment. One can still maintain that identity is a simple relation in the following sense:

"[i]dentity is utterly simple and unproblematic. Everything is identical to itself; nothing is ever identical to anything except itself. There is never any problem about what makes something identical to itself; nothing can ever fail to be. And there is never any problem about what makes two things identical; two thing never can be identical." (Lewis 1986: 192-193)

Here are two ways how: while identity relates one and the same thing to itself, features like those mentioned by Gasser and Stefan belong to a relation that connects distinct person stages (Lewis 1976: 20) or one person to more than one time (Noonan 2003: 88). Whether or not one thinks that the latter relation is analysable in terms of other relations, or reduces to other relations, etc., the former can still be perfectly primitive, unanalysable, or ungrounded. The complex view can accept Lewis' remarks on the simplicity of identity. 


\subsection{Analysability}

As I understand analysability, it denotes a feature of either the meaning of a term or the content of a concept. In the first case, application of the term entails application of one or more further terms. In the second case, one or more constitutive principles of the concept are available. The meaning of 'triangle' entails, for example, 'having three sides'. And the content of triangle is such that it has 'applies to $x$ if $x$ has three sides' as one of its constitutive principles. I see two reasons why rejection of an analysis of personal identity is not the mark of the simple.

Firstly, there is a sense in which paradigm cases of the simple view can be open to there being a term 'personal identity' or a concept personal identity that is analysable: they can accept an analysis of either in terms of, e.g., soul or monad identity.

Secondly, I do not have the impression that the disputants disagree on the status of a term or a concept. They are talking about what is the case at the object level, or the level of facts. The disagreement concerns the status of the things to which terms and concepts can refer, apply or disapply. If so, it is more promising to turn to metaphysical candidate claims.

\subsection{Consist-in Claims}

To understand the simple view as a denial of consist-in claims about personal identity leads to the problems described in Sect. 2.3: some paradigm cases of simplicity are not simple in this sense. If they accept (1), then they do defend consist-in claims about personal identity, although the more basic facts do not concern psychologies or bodies. I take it that analogous remarks apply to attempts to capture the difference in terms of idioms like 'is nothing more' or 'reduces to'.

\subsection{Necessary and Sufficient Conditions}

Gasser and Stefan suggest that simple positions deny necessary and sufficient conditions for personal identity. But again, as described in Sect. 2.3, some simple positions do provide conditions for personal identity. And as I will describe in Sect. 4.2, I see no reason why simple positions should not be able to think of these conditions as necessary, sufficient or both. If so, they are not in disagreement with the complex view about the existence of such conditions.

\subsection{Circularity/Informativeness}

Are these conditions circular or informative? Of course paradigm cases of simplicity deem some paradigmatically complex positions circular. For example, Butler (1736: 100) thinks this way about Locke's account.

A more contemporary circularity charge is levelled by Lowe (2012: 149-151): he paraphrases Neo-Lockeanism as requiring that for person $p$ to be identical to person $q$, any conscious experience had by $p$ at any earlier time is quasi-remembered at least ancestrally by $q$ at any later time, and vice versa. Lowe supposes it is important 
for Neo-Lockeanism that it is one and the same conscious experience which $p$ and $q$ share. He also supposes that any criterion for experiences will make reference to their subjects: $e$ and $f$ are the same experience if and only if $e$ and $f$ are qualitatively indistinguishable and had by the same person at the same time. If so, identity of experiences presupposes personal identity. Neo-Lockeanism is thus circular.

I am not concerned with whether Lowe's criticism is just. It is not obvious to me that Neo-Lockeans require that one and the same experience is shared by $p$ and $q$. Two distinct mental states that stand in appropriate causal relations could be deemed sufficient, too. I would also like to remain non-committal on whether or not a given experience has its subject essentially.

What I would like to note is that although Butler and Lowe level circularity charges against Lockeanism, neither they nor simple positions in general need to deem any account of personal identity circular. For example, Lowe (2012: 151-152) explicitly denies that brain-based complex positions are circular (he rejects them for different reasons $(2012$ : 142, 152)): they take personal identity to consist in brain identity, and brain identity does not presuppose personal identity. He seems to think that Swinburne's position is non-circular for analogous reasons (2012: 151-152). Thus, he deems only some but not all complex positions circular because their proposed criterion of personal identity in fact presupposes personal identity. And at least some simple positions are deemed non-circular because personal identity is defined in terms of entities of a different kind whose identity does not presuppose personal identity. If so, the existence of non-circular conditions of personal identity is not what the complex and the simple view disagree about.

Given Sect. 2.3, this is hardly surprising. We have seen that some simple positions can accept Parfit's condition (2), the possibility of a description of all relevant facts without presupposing the existence or persistence of persons. Such a description would contain non-circular conditions whose obtainment establishes personal identity. Moreover, I certainly would find it informative that personal identity consists in soul identity, and that I go where my soul goes. How I could have epistemic access to where my soul goes is a difficult question, but it concerns a different topic.

I conclude that Gasser and Stefan's account lacks precision and just like Parfit's account fails to capture the paradigm cases.

\section{Noonan}

Noonan has recently provided a new proposal.

\subsection{The Proposal}

Noonan's proposal presupposes that problems of identity over time can be reduced to problems about kind-membership (2003: Ch. 5.3). He suggests:

"the complex view is the view that there are non-trivial, non-redundant, nonidentity-involving diachronic constraints on personhood. The simple view is 
that there are not, that the only non-trivial, non-redundant diachronic constraints on personhood are identity involving." (Noonan 2011: 74)

A diachronic constraint on personhood has the form:

"If $x$ is a person, then if $x$ exists at $t$ and $t^{*}, R x t t^{*}$." (Noonan 2011: 74)

Such a constraint is non-trivial if $R x t t^{*}$ does not logically follow from the antecedent. And it is non-redundant if it is not entailed by the totality of synchronic constraints on personhood, each of which has the form:

"If $x$ is a person, then if $x$ exists at $t, F x t$ " (Noonan 2011: 73),

where $F$ is a property of $x$ that says nothing about any other times than $t$. Finally, a diachronic constraint on personhood is identity-involving if

"its satisfaction requires that something other than a person exists at times $t$ and $t$ *" (Noonan 2011: 74).

Requiring the existence of one and the same soul at the relevant times would be an identity-involving constraint on personhood, provided persons are not souls. However, Noonan (2011: 75) notes that Swinburne's position fits this description and provides a criterion for soul identity over time: identity of form and continuity of immaterial stuff (Swinburne 1984: 27). Continuity of immaterial stuff does not require identity of immaterial stuff, and so Swinburne's position includes a nonidentity-involving constraint on personhood. Noonan's proposal therefore makes Swinburne's position count as complex. This classification is revisionary, but it is not what I will object to in the following.

\subsection{Refinement}

Olson (2012: 56-57) argues that pace Noonan, simple positions accept non-trivial, non-redundant and non-identity-involving diachronic constraints on personhood. Assume with Noonan that any person is essentially a person, and consider the diachronic constraint

No Transformation (NT): if $x$ is a person, then if $x$ exists at $t$ and $t^{*}$, it is not the case that $x$ is wholly material at $t$ and wholly immaterial at $t^{*}$.

This constraint is obviously not trivial or identity-involving. And in order to count as redundant, the diachronic constraint would have to follow from synchronic constraints on personhood. Olson thinks this need not be the case here. We can be neutral on whether

If $x$ is a person, then if $x$ exists at $t, x$ is wholly material,

yet be convinced that $(N T)$ is true. $(N T)$ then would be a non-redundant constraint.

Now, since Noonan defines the simple view as the thesis that the only non-trivial, non-redundant diachronic constraints on personhood are identity-involving, a position that accepts $(N T)$ is not simple. The trouble is, Olson says, that no 
philosopher denies $(N T)$. No one thinks that if you are now wholly material, you can become wholly immaterial (see also Duncan 2014: 286). Thus,

"Noonan's proposal makes the simple view so strong that no one actually holds it" (Olson 2012: 57).

This would be a bad result. The goal was to explain what unites proponents of the simple view, not to declare the simple view unendorsed. However, I see two reasons why Olson's criticism can be resisted.

First reason: Some proponents of the simple view are not neutral on whether persons are material. Reid thinks that persons are monads, and monads are immaterial. He thus accepts $(N T)$, but only as a redundant constraint, i.e., one that follows from synchronic constraints on personhood. Depending on the particular position, similar remarks can apply if persons are taken to be egos or souls. Moreover, it is worth repeating that given Sects. 2.3 and 3.4, I deny that redundancy of this kind makes a position uninformative.

Second reason: Let us grant that $(N T)$-style constraints are not redundant. Olson is still exaggerating when he says that everyone accepts them. For example, Madell, a proponent of the simple view, writes:

"[W]hat unites the experiences in any of these biographies [of one and the same person across possible worlds] is that they belong, unanalysably, to the one mind, and that their doing so is compatible with the absence of objective connection between them." (Madell 1981: 107)

His suggestion seems to be that with respect to the possible history and life of a person, anything goes. The only restriction is that it needs to be her history and life, and this is a brute matter. Thus, Madell will reject (NT). And he is not alone: Chisholm (1969: 138) and Swinburne (1984: 25) offer similar considerations. These positions might seem bold, but it is not my intention to assess whether they are true. What matters for my purposes is that there are simple positions who reject, as Noonan (2003: 94) recognizes, even the sparsest non-redundant constraints.

Olson can still complain that Noonan overgeneralizes from the fact that some or many proponents of the simple view reject (NT)-style constraints to the claim that all simple positions do so. If Olson shows that some accept non-redundant (NT)style constraints without being subject to the First reason described above, Noonan's definition of the simple view would be too narrow.

But even then, I think we can improve Noonan's proposal accordingly. A natural thought is that if complex and simple views need not disagree on the features of available diachronic constraints on personhood, maybe they will differ on sufficient diachronic conditions

If $x$ exists at $t$ and $x$ exists at $t^{*}$ then $R x t t^{*}$, then $\mathrm{x}$ is a person (cf. Noonan 2003: 89).

Presumably, we could define 
Simple*: If there are non-trivial, non-redundant, non-identity-involving diachronic constraints on personhood, $R x t t^{*}$, then joint satisfaction of them does not guarantee that $x$ is a person.

Complex*: There are non-trivial, non-redundant, non-identity-involving diachronic constraints on personhood, joint satisfaction of which guarantees that $x$ is a person.

Simple* is compatible with there being

(I) no constraints at all;

(II) only constraints that are trivial, redundant and/or identity-involving (Noonan's definition of simplicity)

as the conditional will be true if its antecedent is false. If (II), simple positions might even accept that these conditions are sufficient for personal identity. For example, if they think that only persons have souls, they could say:

if $x$ exists at $t$ and $x$ exists at $t^{*}$, then if $x$ at $t$ and $x$ at $t^{*}$ have the same soul, then $x$ is a person.

Of course, one difference in the sufficient conditions specified by the complex and simple view remains:

Simple ${ }_{\text {continued }}$... If there are sufficient diachronic conditions of personhood, these conditions are trivial, redundant, and/or identity-involving.

These modifications meet Olson's objection. I have granted Olson that simple positions can accept non-redundant constraints. But Simple* accommodates Olson's supposition and constitutes only a slight tweak to Noonan's proposal.

\subsection{Being Identity-Involving}

The problem for Noonan's proposal is that being identity-involving is an unsuitable feature to focus on when defining complexity and simplicity. Consider a position according to which persons are not identical to biological lives, yet

Same Biological Life (SBL): if $x$ is a person, then if $x$ exists at $t$ and $t^{*}, x$ has at $t$ the same biological life that $x$ has at $t^{*}$.

Let us assume that the position accepts no other non-identity-involving constraints. Consider the judgements of Noonan's proposal if the position is extended to include one of the following further assumptions:

There are no necessary and sufficient conditions for life identity over time. $(S B L)$ requires the existence of something other than the person at both $t$ and $t^{*}$ : one and the same biological life. Given the assumption, nothing more can be said about when this condition is satisfied. Unlike in the Swinburne case, we cannot unpack a non-identity-involving constraint from $(S B L)$. Noonan's proposal thus classifies the position as simple. 
Mereological Essentialism. $x$ at $t$ can have the same biological life as $x$ at $t^{*}$ only if the life $x$ has at $t$ is composed of exactly the same parts as the life of $x$ at $t^{*}$. Again, $(S B L)$ requires the existence of something other than the person at both $t$ and $t^{*}$ : all of the parts that make up the biological life. No nonidentity-involving constraint can be unpacked. Noonan's proposal classifies the position as simple.

However, without these further assumptions, the position presumably is complex: in order for $x$ to exist at both $t$ and $t^{*}$, likely some kind of continuity of parts rather than their identity is needed between the life $x$ has at $t$ and the life $x$ has at $t^{*}$.

First of all, acceptance of ( $S B L)$ brings a position so close to paradigmatically complex positions (for example Olson 1997: 16-17) that it is unclear why it should be labelled simple even in the wildest circumstances. It would be a stretch to accept this simply as a surprising feature of the proposal.

Secondly, whether or not a constraint is identity-involving turns on a relational property of the constraint: its being accompanied by certain other, external commitments. If extra theory tells an identity-involving story about the entities figuring in $R x t t^{*}$, the position that accepts the constraint is simple (provided no other non-identity-involving constraints have been accepted); if not, it is complex (provided $R x t t^{*}$ is neither trivial nor redundant). But no one has ever thought of the simplicity or complexity of a position as turning on a relational matter in this way. Neither does anyone think that a position turns from complex to simple or vice versa if it extends, contracts, or if its background theory changes. A nice anecdote from Olson (2012: Fn. 9) is telling here: Swinburne nowadays thinks that contrary to what he used to believe, there is no criterion for soul identity over time. But no one, including Swinburne himself, thinks that his position turned from complex to simple. To be fair, no one except Noonan would have classified Swinburne complex in the first place. But suppose we accept $(S B L)$, and later become convinced that, e.g., one of the two further assumptions above holds. According to Noonan, we first held a complex position and ended up with a simple one. Neither this diagnosis nor its grounds are anticipated in the literature.

\section{Two Purposes of a Distinction}

We could stipulate to use terms in this way, but it is another question whether and why this is advisable. When we reflect on the problems of the examined proposals, it seems warranted to take a step back and ask: why are we interested in whether a view is complex or simple?

I see two minimal purposes of having a given distinction in our discourses: Classification and Endorsement. I will now explain them in turn.

Classification. A distinction can help us to classify positions with respect to shared features and to organize them into families of positions. This helps us to get an overview on their similarities and differences, and therewith facilitates navigation in the landscape of positions. 
(i) We can define a distinction and go through existing positions in order to determine whether or not they fall on one side of the distinction. Asserting that a position falls on one side of the distinction describes the position as satisfying certain conditions.

(ii) Alternatively, we start out with a number of positions whom we suspect to share important features, group them provisionally, and then think further about what unites them. Given the clear picture of which positions are paradigm cases, this seems to be what we are doing in the case of the complex versus simple distinction.

Regarding Classification, Parfit's and Gasser and Stefan's definitions do not do a good job. Their conditions for complexity are jointly satisfied by paradigm cases of simplicity. There is a mismatch between the positions we antecedently group together and what follows from the definitions. Moreover, the definitions include imprecisions and shifts in key notions. Whether a given position lands on one side of the distinction depends on which notion the definition picks out. And we have seen (Sects. 3.1-3.4) that no specification gives us the right results.

Setting Swinburne aside, Noonan's account classifies extant paradigm cases reasonably well. However, its sensitivity to arguably irrelevant external commitments due to the relational nature of being identity-involving raises doubts about the grounds for these classifications. For the purpose of (ii)-style Classification, not just any feature shared by the positions will interest us. We are looking for features that are both shared by the positions and constitutive for each of them. Here is an analogy. Suppose we live in a world where all round things happen to be red. It would be too quick to conclude that the difference between round and non-round things has something to do with redness. We should come to see that round things could be blue, transparent, etc., and so that facts about roundness can float free of facts about colours. The positions discussed in Sect. 4.3 are the analogues to these counterexamples. They show that even if most paradigm cases of complexity and simplicity respectively share the features Noonan's proposal mentions, serious worries remain whether possession of these features is distinctive of the respective view. Amongst others, we can thus remain sceptical whether the proposal can deal with the classification of new positions, and how it behaves once interpretation proceeds and gradually uncovers further commitments of a given author.

The difficulties encountered in the previous sections show that it is hard to see which features paradigmatic instances of complexity or simplicity share in the sense just described. The most charitable thing to say is that 'complex' and 'simple' try to stand in for more specific features. The problem is that it is not clear which ones these are. Given the failure of the examined proposals, there should be a significant amount of pessimism and no optimism about the availability of an account.

Classification of positions is not the only purpose of having a distinction:

Endorsement. A distinction can help us to express our thoughts through endorsement of one of the sides. Distinguishing one kind of view from another provides us with targets for affirmation and criticism. Again, there are at least two possible directions: 
(iii) We may find a number of positions independently plausible (or implausible). If we learn from Classification that they all fall on one side of a relevant distinction, and if we know of no implausible (or plausible) position that falls on the same side, our affirmation (or criticism) can extend beyond the individual cases and to the side of the distinction as such.

(iv) Alternatively, upon reflection on the distinction in abstraction from particular instances of either side, we become convinced that one side is correct, and therefore become sympathetic to its instances.

Suppose we endorse the complex view as in (iv). Because of the difficulties in Classification, this endorsement should strike us as odd. Complexity is due to the possession of features as specified in Classification. Given that this specification was unsuccessful, it is not clear what we have endorsed now. It is thus unclear on what grounds we made the endorsement and whether these grounds are reasonable. After all, we can hardly give reasons for the affirmation of a view if we cannot explain what the view is. Instead, (iv)-style Endorsement will only be plausible derivatively upon plausible affirmation of features we ascribe to the view.

Nothing seems wrong with (iii)-style Endorsement of the complex or the simple view. Indeed (iii)-style rejections of the simple view are common: it is often dismissed on the grounds that simple positions' stances on marks of complexity as defined by Parfit, Gasser and Stefan, and others are coherent but implausible (Shoemaker 1985: 449; Duncan 2014: 291, 293-294). However, if what I argued is right, then these dismissals proceed on the wrong grounds. As shown in Sects. 2 and 3 , simple positions can accept conditions which many take to be constitutive of the complex view. If so, further explanation and interpretation are needed to bring out what if anything is wrong with the simple view.

Further explanation is also needed on how supposed theoretical or practical consequences follow from endorsement of either side of the complex versus simple distinction. For example, Parfit endorses the complex view and thinks it follows that identity is not what matters. Of course, he defends this latter claim not merely because he endorses the complex view (whatever that means), but because he thinks conditions like (1) and (2) obtain (1984: 260-263). He happens to think that acceptance of these conditions is what unites proponents of complexity. But this classification is not only unimportant, as the practical conclusions are thought to follow from conditions like (1) and (2) already; it is also mistaken. (1) and (2) can be accepted by simple positions. If there really is a difference in practical judgements between the complex and the simple view, more needs to be said on why this should be the case. (1) and (2) as well as the other conditions mentioned by the proposals cannot ground the asymmetry, because the complex and the simple view are not in disagreement about them.

Analogous remarks apply to the claim that the complex view cannot justify future-directed self-concern, or cannot justify it to the extent that the simple view can (Parfit 1984: 307-312). Some take this as a reductio of the complex view and a 
reason to endorse the simple view (Butler 1736: 102, 104. Madell 1981: 109-112. Swinburne 1974: 246), others as a reason for revision of ordinary patterns of concern that presuppose the simple view (Parfit 1984: 280). But we have not yet seen a satisfying account of the difference between the complex and the simple view, let alone one that licenses these claims.

\section{Conclusion}

From the assumption that there is an important difference between complex and simple positions, authors have drawn conclusions about what matters, whether special concern for future selves can be justified, whether persons can undergo fission, and which theory of personal identity is true (Parfit 1984; Shoemaker and Swinburne 1984; Shoemaker 2012; Swinburne 2012; Madell 1981, 2015). But if I am right, taking sides in the complex versus simple distinction cannot play a role in these disagreements. This is because as shown, authors largely agree on the conditions discussed in the present paper, yet disagree on the topics just mentioned.

Specifically, we get a new, straightforward reason for why we can discard claims according to which taking sides in the complex versus simple distinction should commit one to a certain stance about what matters: conditions like Parfit's (1) and (2) supposedly establish that contrary to what simple positions and common sense suggest, identity is not what matters. In response, not only can we point at authors who think that metaphysical insights, including insights on the nature of personal identity, concern the fundamental structure of reality and are thus neutral on ordinary facts of practical relevance (Sider 2012; Olson 2012: 81), or that the relation between metaphysics and practical concerns is at least much less straightforward than Parfit's revisionism suggests (Shoemaker 2007). And not only can we point at familiar considerations of authors like Lewis (1976) who describe how a position can accept the conditions taken to be constitutive of the complex view and still be in perfect agreement with the proponents of the simple view regarding what matters and whether a person can divide. Given the present paper, we can make a new point: conditions like (1) and (2) are satisfied by paradigmatically simple positions all along. Stances on these conditions alone thus cannot be what grounds differences in practical judgements.

Finally, the complex versus simple distinction turned out to be unhelpful with respect to Classification, and Endorsement should take place on the basis of more specific features anyways. If not in terms of complexity and simplicity, how should we frame debates about personal identity? I recommend speaking in terms of the specific conditions and related concepts for which the terms 'complex' and 'simple' try to stand in. Promising candidate conditions presumably make positive or negative claims regarding the groundedness, criteriality, materiality, fundamentality, and/or the mereological status of persons and their persistence. It has been argued in this paper that the prospects for providing an account of the complex versus simple distinction are bad, and so we will likely arrive at a more fragmented landscape of views. But this fragmentation will be outweighed by benefits in clarity and precision. 
Acknowledgments Many thanks to Steinvör Thöll Árnadóttir, Lorianna Clarke-Alexander, Patrick Greenough, Bruno Jacinto, Hasen Khudairi, Poppy Mankowitz, Ravi Thakral, Caroline Torpe Touborg, Wolfgang Sattler, and audiences at the Universities of St Andrews (Arché Metaphysics Research Group and Postgraduate Friday Seminar), Milan (Conference "Me, the Self, and I"), Zurich (Workshop with Udo Thiel) as well as VU Amsterdam (OZSW Conference) for helpful comments on ideas from this paper. Special thanks to Katherine Hawley for frequent and invaluable discussion, guidance, and encouragement. Two anonymous referees provided insightful comments and suggestions that helped me to improve the paper in numerous ways. I am grateful for a scholarship from the German Foreign Exchange Service (DAAD) which I received while this paper was written.

Open Access This article is distributed under the terms of the Creative Commons Attribution 4.0 International License (http://creativecommons.org/licenses/by/4.0/), which permits unrestricted use, distribution, and reproduction in any medium, provided you give appropriate credit to the original author(s) and the source, provide a link to the Creative Commons license, and indicate if changes were made.

\section{References}

Butler, J. (1736). Of personal identity. In J. Perry (Eds.), Personal identity (pp. 99-105). Berkeley: University of California Press, 1975.

Chisholm, R. (1969). The loose and popular and the strict and philosophical senses of identity. In N. S. Care \& R. H. Grimm (Eds.), Perception and personal identity. Cleveland: The Press of Case Western Reserve University.

Duncan, M. (2014). A challenge to anti-criterialism. Erkenntnis, 79, 283-296.

Garrett, B. (2006). What is this thing called metaphysics?. London and New York: Routledge.

Gasser, G., \& Stefan, M. (2012). Introduction. In G. Gasser \& M. Stefan (Eds.), Personal identity: Complex or simple? (pp. 1-17). Cambridge: Cambridge University Press.

Lewis, D. K. (1976). Survival and identity. In A. O. Rorty (Ed.), The identities of persons (pp. 17-40). Berkeley: University of California Press.

Lewis, D. K. (1986). On the plurality of worlds. Cambridge: Cambridge University Press.

Lowe, E. J. (2012). The probable simplicity of personal identity. In G. Gasser \& M. Stefan (Eds.), Personal identity: Complex or simple? (pp. 137-155). Cambridge: Cambridge University Press.

Madell, G. (1981). The identity of the self. Edinburgh: Edinburgh University Press.

Madell, G. (2015). The essence of the self. In defense of the simple view of personal identity. Abingdon and New York: Routledge.

Noonan, H. (2003). Personal identity (2nd ed.). London and New York: Routledge.

Noonan, H. (2011). The complex and simple views of personal identity. Analysis, 71(1), 72-77.

Olson, E. T. (1997). The human animal. Oxford: Oxford University Press, 1999.

Olson, E. T. (2012). In search of the simple view. In G. Gasser \& M. Stefan (Eds.), Personal identity: Complex or simple? (pp. 44-62). Cambridge: Cambridge University Press.

Parfit, D. (1982). Personal identity and rationality. Synthese, 53, 227-241.

Parfit, D. (1984). Reasons and persons. Oxford: Clarendon Press, 1987.

Parfit, D. (1995). The unimportance of identity. In H. Harris (Ed.), Identity: Essays based on Herbert Spencer Lectures given in the University of Oxford (pp. 13-45). New York: Clarendon Press.

Reid, T. (1785a). Of identity. In J. Perry (Ed.), Personal identity (pp. 107-112). Berkeley: University of California Press, 1975.

Reid, T. (1785b). Of Mr. Locke's account of our personal identity. In J. Perry (Ed.), Personal identity (pp. 113-118). Berkeley: University of California Press, 1975.

Shoemaker, D. (2007). Personal identity and practical concerns. Mind, 116(462), 317-357.

Shoemaker, S. (1985). Critical notices. Mind, 94(365), 443-453.

Shoemaker, S. (2012). Against simplicity. In G. Gasser \& M. Stefan (Eds.), Personal identity: Complex or simple? (pp. 123-136). Cambridge: Cambridge University Press.

Shoemaker, S., \& Swinburne, R. (1984). Personal identity. Oxford: Blackwell. 
Sider, T. (2012). The evil of death: What can metaphysics contribute? In B. Bradley, F. Feldman \& J. Johansson (Eds.), The Oxford handbook of philosophy of death (pp. 155-166). Oxford: Oxford University Press.

Swinburne, R. (1974). Personal identity. Proceedings of the Aristotelian Society, 74, 231-247.

Swinburne, R. (1984). Personal identity: The dualist theory. In S. Shoemaker \& R. Swinburne (Eds.), Personal identity (pp. 1-66). Oxford: Blackwell.

Swinburne, R. (2012). How to determine which is the true theory of personal identity. In G. Gasser \& M. Stefan (Eds.), Personal identity: Complex or simple? (pp. 102-122). Cambridge: Cambridge University Press.

Wasserman, R. (2012). Personal identity, indeterminacy, and obligation. In G. Gasser \& M. Stefan (Eds.), Personal identity: Complex or simple? (pp. 63-81). Cambridge: Cambridge University Press.

Zimmerman, D. (2012). Materialism, dualism, and "simple" theories of personal identity. In G. Gasser \& M. Stefan (Eds.), Personal identity: Complex or simple? (pp. 206-235). Cambridge: Cambridge University Press. 